๑ Entomologica Fennica. 19 September 2003

\title{
Platygastridae from the Canary Islands and Madeira (Hymenoptera: Platygastroidea)
}

\author{
Peter Neerup Buhl \& Martti Koponen
}

Buhl, P. N. \& Koponen, M. 2003: Platygastridae from the Canary Islands and Madeira (Hymenoptera: Platygastroidea). — Entomol. Fennica 14: 105-108.

Distributional and phenological records are given for 22 platygastrid species from the Canary Islands and Madeira.

Peter Neerup Buhl, Troldhфjvej 3, DK-3310 Ølsted, Denmark; E-mail: pnbuhl@zmuc.ku.dk

Martti Koponen, Department of Applied Biology, P.O.Box 27, FIN-00014

University of Helsinki,Finland; E-mail: martti.a.koponen@helsinki.fi

Received 30 May 2002, accepted 5 March 2003

\section{Introduction}

Hitherto only very few records of Platygastridae from the Canary Islands and Madeira have been published. Of the seven species recorded from Madeira by Graham (1984) only one was determined to species. Buhl (2001) described one new species of Platygaster from Tenerife, and Buhl (2003) another new species of Platygaster and one of Synopeas, also from the Canaries.

In this paper, records of 22 species are given, mostly from the recent collections of M. Koponen and M. Báez. It appears that many species known from NW Europe are also present on the Canary Islands and Madeira, but that on these islands they appear as adults most of the year (i.e. they have more generations a year than in NW Europe).

Abbreviations: $\mathrm{G}=\mathrm{La}$ Gomera, $\mathrm{M}=$ Madeira, $\mathrm{T}=$ Tenerife, $\mathrm{P}=$ La Palma; $\mathrm{MK}=\mathrm{M}$. Koponen det.; PNB = P. N. Buhl det.; DABH = Department of Applied Biology, University of Helsinki; TFMC $=$ Museo de Ciencias Naturales de Tenerife; $\mathrm{MNCN}=$ Museo Nacional Ciencias Naturales, CSIC, Madrid.

\section{Records of Canarian and Madeiran Platygastridae}

Allotropa conventus Maneval, 1936

M: Funchal, Preces - Faja (300 m a.s.1.), 11.XII.1994, 1 ㅇ (PNB, DABH).

\section{Allotropa sp.}

G: Antoncojo, 26.III.1999, 25 ㅇ (MK). T: 2 km W Buenavista, 8.IV.1993, 4 ㅇ (MK). Santa Úrsula, La Quinta, 28.III.1993, 3 ㅇ, 29.III.1993, 3 ㅇ, 4.IV.1993, 4 ㅇ, 5.IV.1993, 1 우 (MK, DABH).

\section{Ambyaspis prorsa (Walker, 1835)}

G: Barranquillos, 18.IX.1977, 1 \% (M. Báez, MK, TFMC). Las Mesetas, 13.VIII.1977, 1 \& (M. Báez leg., MK, TFMC). Los Gallos, 14.IX.1977, 1 ㅇ (M. Báez leg., MK, TFMC). P: National Park of Caldera de Taburiente, 3.VIII.-22.IX.1999, 23 우 
(MNCN, PNB). T: Pinar. La Guancha, 11.VIII.1996, 3 ㅇ (E. Guerrero leg., MK, TFMC). Ijuana, 30.IX.-4.X.1989, 3 ㅇ, 8.IX.1985, 1 Ơ (M. Báez leg., PNB, TFMC). Las Agras, 5.XII.1986, 1 ㅇ (M. Báez leg., PNB, TFMC).

Note. New to La Gomera and Tenerife.

\section{Amitus longicornis (Förster, 1878)}

M: Estreito de Camara de Lobos (550 m a.s.1.), 13.XII.1995, 15 Q (MK, DABH, TFMC). Funchal, Amparo (150 m a.s.l.), 20.XII.1994 (MK, DABH); Preces — Faja (300 m a.s.1.), 11.XII.1994, 1 ㅇ (MK, DABH); Quebradas, 11.XII.1995, 1 \% (MK, DABH). Monte (550-750 m a.s.1.), 17.XII.1994, 7 ㅇ 1 ơ (PNB, DABH, TFMC). T: Barlovento, 20.IX.1998, 2 우 (E. Guerrero leg., MK, TFMC). Note. New to Canary Islands.

\section{Inostemma boscii (Jurine, 1807)}

T: $2 \mathrm{~km}$ W of Buenavista, 8.IV.1993, 1 우 1 O" (MK, TFMC). La Orotava, El Rincon, 1.XII.2002, $10^{7}(\mathrm{MK}, \mathrm{DABH})$. Puerto de la Cruz, Parque Taoro, 30.III.1993, 1 우 (MK), 20.XII.1997, 1 우 1 ơ; 2.IV.1999, 1 O' (PNB, DABH).

\section{Inostemma $\mathbf{n} . \mathbf{s p}$.}

P: Los Sauces (380-475 m a.s.l.), 7.IV.1998, 1 ㅇ (PNB, DABH).

\section{Iphitrachelus gracilis Masner, 1957}

P: National Park of Caldera de Taburiente, 22.IX.1999, 1 ㅇ 1 O' (PNB, MNCN).

\section{Leptacis ozines (Walker, 1835)}

M: Funchal. Monte (550 m a.s.l.), 3.XI.1996, 41 O (MK, DABH, TFMC); Old Monte Gardens, 5.XI.1996, 1 Q (PNB). Vale do Paraiso, 6.XI.1996, 5 우 (PNB) + 2 우 (MK), 7.XI.1996, 4 ㅇ 1 ơ (PNB, DABH). P: El Paso, Cumbre Nueva (870-908 m

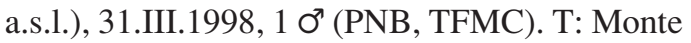

Los Silos, 14.XII.1997, 1 O’. Taberno, 12.I.1998, 1 ㅇ (M. Báez leg., PNB, TFMC).

Note. Probably identical to "Leptacis sp. near tipulae (Kirby)" mentioned by Graham (1984).

\section{Leptacis vlugi Buhl, 1997}

M: Funchal. Monte (550 m a.s.1.), 3.XI.1996, 3 ㅇ (MK); Old Monte Gardens, 5.XI.1996, 3 \& (PNB). Vale do Paraiso, 7.XI.1996, 2 \& 2 O" (PNB) + $10^{7}$ (MK, DABH). P: Barlovento, Laguna do B. (732 m a.s.1.), 25.VII.1997, 1 ᄋ (MK, DABH). Breña Baja, Los Cancajos, 22.II.1997, 1 우 (MK); 4.III.1997, 1 우 (PNB, DABH); 29.III.1998, 5 ㅇ 1 O'(PNB, DABH, TFMC). National Park of Caldera de Taburiente (PNB, MNCN). T: Aguamansa(1000-1400 m a.s.l.), 6.IV.1993, 3 ơ (PNB); 12.XII.1997, 2 우 (MK, DABH). $2 \mathrm{~km}$ W of Buenavista, 8.IV.1993, 1 ㅇ (PNB, TFMC), 1 \& (MK). Puerto de la Cruz, Parque Tajunaste, 1.IV.1999, 1 \& (MK, DABH). Santa Úrsula, La Quinta, 28.III.1993, 1 ᄋ (PNB, TFMC); 29.III.1993, 1 ㅇ (PNB, DABH); 7.IV.1993, 2 우 (PNB, DABH, TFMC).

\section{Platygaster baezi Buhl, 2003}

T: Punta de Teno, 14.XII.1997, 3 Q 1 O’ (M. Báez leg., PNB, TFMC, one $Q$ in ZMUC).

\section{Platygaster munki Buhl, 1994}

P: Breña Baja, Los Cancajos, 29.III.1998, 1 Ơ (MK),

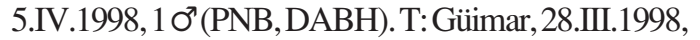
2 ㅇ (E. Guerrero leg., MK, TFMC). Puerto de la Cruz, Parque Taoro, 2.IV.1999, 6 O’ (MK, DABH, TFMC).

\section{Platygaster nisus Walker, 1835}

T: Aguamansa, 12.XII.1997, 1 ㅇ (MK, TFMC). Punta de Teno, 14.XII.1997, 10 ㅇ 3 ơ (PNB, DABH, TFMC).

\section{Platygaster cf. pedasus Walker, 1835}

T: 2 km W Buenavista, 8.IV.1993, 1 (MK). Puerto 
de la Cruz, Parque Taoro, 15.XI.2000, 1 \& (MK). Punta de Teno, 14.XII.1997, 1 \& (PNB, DABH).

\section{Platygaster tenerifensis Buhl, 2001}

M: Monte (550-750 m a.s.1.), 17.XII.1994, 1 우 (PNB, TFMC). Vale do Paraiso (740 m a.s.l.), 6.XI.1996, 1 \& (MK, DABH). P: Barlovento, 25.II.1997, 1 ᄋ (MK). Breña Baja, Los Cancajos, 22.II.1997, 3 9 (MK, TFMC); 2.III.1997, 3 ᄋ 1 o" (MK, DABH); 4.III.1997, 1 \% 1 ठ7' (PNB, TFMC); 29.III.1998, 1 ㅇ 4 O $^{\prime}$ (MK, DABH); Monte Breña, 27.II.1997, 12 ९ (PNB, DABH) + 1 ○" (MK); 30.III.1998, 8 ㅇ 4 O' (MK, DABH). El Paso, Cumbre Nueva (870-908 m a.s.1.), 31.III.1998, 2 ㅇ (MK, TFMC). Los Sauces, 7.IV.1998, 1 웅 (PNB, TFMC). T: El Seuzal, 10.VII.1997, 1 ㅇ (E. Guerrero leg., MK, TFMC). La Laguna, 11.XII.1997, 1 O (MK, TFMC). La Orotava, El Rincon, 1.XII.2002, $10^{7}$ (MK, DABH). Puerto de la Cruz, Parque Taoro, 19.XII.1997, 2 O' (PNB, DABH, TFMC); 20.XII.1997, 1 ㅇ 1 0" (MK, TFMC). Santa Úrsula, La Quinta, 4.XII.1992, 3 ㅇ (DABH, TFMC); 28.III.1993, 1 ㅇ (TFMC); 30.III.1993, 6 ㅇ (TFMC); 1.IV.1993, 27 ㅇ (TFMC); 3.IV.1993, 8 ○ (TFMC); 4.IV.1993, 1 O (TFMC); 5.IV.1993, 12 ९ (TFMC); 7.IV.1993, 23 ○ $10^{7}$ (DABH, TFMC); 9.IV.1993, 10 ㅇ (PNB, TFMC); 8.XII.1993, 2 \% (MK, DABH); 10.XII.1993, 1 ㅇ (MK, DABH). Tegueste, 4.VIII.1933, 1 ᄋ (? leg., MK, TFMC). Mte. Aguirre 11.VI.1985, 10.XI.1985, 26.II.1986, 1 우 3 ơ; Las Agras 5.XI.1986, 1 ㅇ. Mte. Los Silos 12.VI.1985, 14.XII.1997, 2 ○ 1 ơ. Palo Blanco 27.VI.1984, 29.XI.1985, 3 \& 1 ơ. Bco. Ijrana 4.X.1984, 1 O$^{7}$. Genores 15.II.1985, 2 Q. Teno Bajo 22.III.1999, 1 ㅇ. (M. Báez leg., PNB, TFMC).

\section{Pseudaphanomerus hyalinatus Szelényi, 1941}

P: El Charco, 28.V.1984 1 ㅇ (M. Báez leg., PNB, TFMC).

\section{Synopeas ciliatus Thomson, 1859}

M: Monte (550-745 m a.s.1.), 17.XII.1994, 1 우 (PNB, DABH); 3.XI.1996, 3 \& (MK, DABH).
Vale do Paraiso, 7.XI.1996, 6 \& (PNB) + 1 \& (MK, DABH). P: Breña Baja, Los Cancajos, 2.III.1997, 1 ㅇ (PNB, TFMC) + 1 ㅇ (MK); 29.III.1998, 3 우 $(\mathrm{PNB}, \mathrm{TFMC})+5$ ९ $(\mathrm{MK})$; Monte Breña, 30.III.1998, 1 ㅇ (MK, DABH). Los Sauces, 7.IV.1998, 1 ㅇ (MK, TFMC). National Park of Caldera de Taburiente (PNB, MNCN). T: Bco. S. Andres, 6.II.1987, 1 Ơ (M. Báez leg., PNB, TFMC). Aguamansa (1000-1400 m a.s.1.), 12.XII.1997, 2 ( (MK, DABH). 2 km W Buenavista, 8.IV.1993, 2 \% (PNB, TFMC) + 9 \% (MK, DABH). Puerto de la Cruz, Barranco San Felipe, 25.XI.2000, 11 ㅇ (MK, DABH); Parque Taoro, 2.IV.1999, 1 ㅇ (MK, DABH). Santa Úrsula, La Quinta, 1.IV.1993, 2 9 (PNB, TFMC); 3.IV.1993, 3 Q (PNB, DABH, TFMC); 5.IV.1993, 5 \% (PNB, TFMC); 7.IV.1993, 6 ९ (PNB, DABH); 9.IV.1993, 3 \% (PNB, TFMC); 10.XII.1993, 1 ㅇ (MK, DABH).

\section{Synopeas dentiscutum (Szabó, 1981)}

Fuerteventura: El Cardón, 28.II.1990, 1 (M. Báez leg., PNB, TFMC).

\section{Synopeas koponeni Buhl, 2003}

P: Breña Baja, Los Cancajos, 27.III.1998, 1 우 (MK, DABH); 5.IV.1998, 1 \% (holotype, PNB, TFMC). T: Güimar, 19.IV.1998, 2 ㅇ (E. Guerrero leg., MK, TFMC). Punta de Teno, 14.XII.1997, 1 ○ (paratype, DABH).

\section{Synopeas sp. nr. koponeni Buhl, 2003}

T: Las Aguas, 8.XII.2002, 1 ㅇ (DABH, MK). Abdomen longer than in S. koponeni.

\section{Synopeas sp. 1}

P: Breña Baja, Los Cancajos, 2.III.1997, 2 우 (PNB, DABH, 4.III.1997, 3 \&, 4.IV.1998, 2 ㅇ (PNB, DABH). Los Sauces (380-475 m a.s.l.) 7.IV.1998, 3 9 (PNB, TFMC). T: 2 km W Buenavista, 8.IV.1993, 7 ( (PNB, DABH, TFMC). Santa Úrsula, La Quinta, 1.IV.1993, 1 ㅇ, 3.IV.1993, 4 
우 $(\mathrm{PNB})+1(\mathrm{MK}) ;$ 5.IV.1993, 4 우 (PNB); 7.IV.1993, 7 ㅇ (PNB, DABH).

\section{Synopeas sp. 2}

M: Jardim da Serra (800 m a.s.1.), 10.XII.1991, 2 O (MK, DABH). P: Barlovento, Laguna de B. (732 m a.s.l.), 25.II.1997, 1 우 (MK, DABH). Breña Baja, Los Cancajos, 22.II.1997, 5 O (MK, DABH), 2.III.1997, 1 \& (PNB, TFMC) + 5 ㅇ (MK, DABH), 4.III.1997, 3 \& (PNB, DABH); Monte Breña, 30.III.1998, 25 \& (MK, DABH). El Paso, Cumbre Nueva (870-908 m a.s.1.), 31.III.1998, 22 \& (MK, DABH). Los Sauces (380-475 m a.s.1.), 7.IV.1998, 6 9 (PNB, DABH, TFMC). T: Aguamansa, 6.IV.1993, 6.IV.1993, 1 \& (PNB). Anaga, Vueltas de Taganana, 24.III.1999, 1 \& (MK). $2 \mathrm{~km} \mathrm{~W}$ Buenavista, 8.IV.1993, 3 ㅇ (PNB) + 3 (MK). La Vega, 23.III.1999, 1 ( $($ MK). Puerto de la Cruz, Parque Taoro, 15.XI.2000, 1 \& (MK). Santa Úrsula, La
Quinta, 28.III.1993, 1 , 1.IV.1993,2 9 , 7.IV.1993, 3 ㅇ, 9.IV.1993, 3 (PNB, DABH).

\section{Synopeas sp. 3}

P: Breña Baja, Los Cancajos, 4.IV.1998, 1 \& (PNB, DABH).

T: 2 km W Buenavista, 8.IV.1993, 2 요 (PNB, DABH, TFMC +2 \& (MK, DABH). Puerto de la Cruz, Taoro, 29.III.1993, 1 \& (PNB, DABH).

\section{References}

Buhl,P.N. 2001: Elevennew species of Platygastrinae(Hymenoptera: Platygastridae). — Folia Ent. Hung. 62: 133-149.

Buhl, P. N. 2003: New or little known Palaearctic species of Platygastrinae (Hymenoptera: Platygastridae). Entomol. Fennica 14: 109-117.

Graham, M. W. R. de V. 1984: Madeira Insects, mainly Hymenoptera Proctotrupoidea, Ceraphronoidea, and Bethyloidea. - Bol. Mus. Mun. Funchal 36: 83-110. 